\title{
A rapid and feasible tool for clinical decision making in community-dwelling patients with COVID-19 and those admitted to emergency departments: the Braden-LDH-HorowITZ Assessment-BLITZ
}

\author{
Erik Lagolio ${ }^{1}$. Jacopo Demurtas ${ }^{2}(-) \cdot$ Roberto Buzzetti $^{3} \cdot$ Giorgio Cortassa $^{1} \cdot$ Stefania Bottone $^{1} \cdot$ Laura Spadafora $^{1}$. \\ Cristina Cocino ${ }^{1} \cdot$ Lee Smith $^{4} \cdot$ Thomas Benzing $^{5,6} \cdot$ Maria Cristina Polidori $^{5,6}$
}

Received: 11 February 2021 / Accepted: 9 July 2021 / Published online: 28 July 2021

(c) Società Italiana di Medicina Interna (SIMI) 2021

\begin{abstract}
There is no univocal standardized strategy to predict outcomes and stratify risk of SARS-CoV-2 infected patients, notably in emergency departments. Our aim is to develop an accurate indicator of adverse outcomes based on a retrospective analysis of a COVID-19 database established at the Emergency Department (ED) of a North-Italian hospital during the first wave of SARS-CoV-2 infection. Laboratory, clinical, psychosocial and functional characteristics including those obtained from the Braden Scale-a standardized scale to quantify the risk of pressure sores which takes into account aspects of sensory perception, activity, mobility and nutrition-from the records of 117 consecutive patients with swab-positive COVID-19 disease admitted to the Emergency Medicine ward between March 1, 2020 and April 15, 2020 were included in the analysis. Adverse outcomes included admission to the Intensive Care Unit (ICU) and in-hospital death. Among the parameters collected, the highest cutoff sensitivity and specificity scores to best predict adverse outcomes were displayed by lactate dehydrogenase (LDH) blood value at admission $>439 \mathrm{U} / \mathrm{L}$, Horowitz Index (P/F Ratio $)<257$ and Braden score $<18$. The estimation power reached 93.6\%. We named the assessment BLITZ (Braden-LDH-HorowITZ). Despite the retrospective and preliminary nature of the data, a multidimensional tool to assess overall functions, not chronological age, produced the highest prediction power for poor outcomes in relation to SARS-CoV-2 infection. Further analyses are now needed to establish meaningful correlations between ventilation therapies and multidimensional frailty as assessed by ad-hoc validated and standardized tools.
\end{abstract}

Keywords COVID-19 · Frailty · Prognostic assessment · Emergency

\section{Introduction}

E. Lagolio and J. Demurtas are equal contributors.

T. Benzing and M.C. Polidori are equal supervisors.

Jacopo Demurtas

eritrox7@gmail.com

1 Emergency Medicine (A\&E), As12 - Hospital Santa Corona, Pietra Ligure, Italy

2 Clinical and Experimental Medicine PhD Program, University of Modena and Reggio Emilia, Strada Casal Nuovo, 2058011 Capalbio (GR), Modena, Italy

3 Clinical Epidemiologist, Bergamo, Italy

4 The Cambridge Centre for Sport and Exercise Sciences, Anglia Ruskin University, Cambridge, UK
The current COVID-19 pandemic has highest fatality rates among the older generations and is exhausting world economies and solidarity [1]. Indeed, vast literature is now

5 Ageing Clinical Research, Department of Internal Medicine and Center for Molecular Medicine, Cologne, University of CologneFaculty of Medicine and University Hospital Cologne, Cologne, Germany

6 Cologne Excellence Cluster on Cellular Stress-Responses in Aging-Associated Diseases (CECAD), University of Cologne, Faculty of Medicine and University Hospital Cologne, Cologne, Germany 
available regarding the infection and protection mechanisms with respect to the Severe Acute Respiratory Syndrome CoronaVirus-2 (SARS-CoV-2). However, it is unclear how long protective immunity lasts and what are safe therapy and immunization protocols for this novel infection [2]. Especially until these critical questions are solved, and in light of the lack of resilience of already overburdened health care systems that cannot offer sufficient and adequate respiratory support and intensive care, a reliable patient's risk stratification and triaging for clinical decision making represents currently the number one healthcare priority in real life [3]. Nevertheless, no standard of care is available to date [4]. In addition, social distancing measures are having a strongly detrimental impact on physical and mental health [5]. Therefore, protecting those at most risk of dying from COVID-19 while relaxing the strictures on others provides a way forward in the SARS-CoV-2 epidemic. A coherent risk stratification algorithm is urgently needed even in the presence of effective vaccination, given the virus is unlikely to disappear in the foreseeable future [6]. Emerging evidence throughout the course of the pandemic has shown associations of age, sex, certain comorbidities, smoking habit, ethnicity, and obesity with adverse COVID-19 outcomes such as hospital admission or death [7, 8]. However, the large heterogeneity especially of the older population hinders the univocal approach to triaging so far.

The aim of the present analysis was to identify indicators of adverse outcomes of COVID-19 using the database of the Emergency Department (ED) of a North-Italian hospital.

\section{Methods}

Records from 117 consecutive patients admitted to the Emergency Room (ER) of Pietra Ligure Santa Corona Hospital from March 1st to April 15th 2020 and identified as COVID-19-positive in the presence of clinical symptoms and by a positive real-time reverse transcriptase-polymerase chain reaction (RT-PCR) nasopharyngeal swab test as well as by radiological diagnosis and clinical criteria were included in this retrospective analysis.

All patients were transferred from the ER to the ED of the same hospital and all of them were admitted to ward. Laboratory, clinical, psychosocial and functional characteristics including those obtained from the Conley Scale-a six-item scale that aims to identify patients at risk of falling — and the Braden Scale—a standardized scale to quantify the risk of pressure sores which takes into account aspects of sensory perception, activity, mobility and nutrition-were performed on admission in all patients. No exclusion criteria were applied to the datasets and no "Do Not Resuscitate" (DNR) patient was present in the sample analyzed. Ethical approval was not required due to the retrospective nature of the work and the entailed use of anonymized routinely collected data. Data analysis approval was obtained by the local governance.

\section{Statistical analysis}

General characteristics were reported as proportions and min, max, median, quartiles, mean as well as standard deviation for categorical and continuous variables, respectively. For the above cited purpose of the investigation, three disease adverse outcomes were chosen on a first step: admission on ICU, invasive ventilation [IV-ventilation forms requiring intubation including invasive continuous positive airway pressure (CPAP) therapy and biphasic positive airway pressure (BPAP)] as well as in-hospital death. Secondly, the significance of the association between each of the 117 independent variables and each of the 3 selected outcomes was tested. To do so, Chi-square testing or Fisher exact test were used in $2 \times 2$ contingency tables to evaluate associations $($ alpha $=0.05)$. Numeric variables were transformed into binary values $(\geq$ median $=1,<$ median $=0)$. To test the weight of a more simplified outcome with high utilization potential across settings technology, IV was excluded and in-hospital death OR admission to ICU were chosen as composite outcome. Third and lastly, the variables found to be associated with either admission on ICU, IV or in-hospital death were tested on the new composite outcome (death OR ICU) followed by dichotomic transformation and multiple logistic regression analysis for those showing significant associations. For each of the independent numerical variables, a ROC analysis was carried out to search for the best cutoff. To do so, the risk of outcome within the quartiles of the variables after binary conversion and best cutoff value identification as a threshold for each was analyzed.

Missing data were replaced by statistical estimates with Mode as imputation value. A comparison with the next neighbor value technique (Last Observation Carried Forward) after list-wise deletion was carried out to confirm the results [9]. To estimate the risks associated to the outcome of interest, a model equation for each of the combinations of the presence/absence of each of the predictive variables was applied. Two-sided alternatives with a significance level of alpha $=0.05$ were considered for all the tests. XLSTAT software was used.

\section{Results}

\section{Study population}

As reported in Table 1, data from 117 patients admitted consecutively to the emergency ward between March 1, 2020 and April 15, 2020 (70 men, mean age $73.1 \pm 14.4$ years) 
Table 1 Characteristics of the sample

\begin{tabular}{llllll}
\hline & $N($ total 117) & $\%$ & Mean (DS) & Median & Min-max \\
\hline Female & 47 & 40.1 & & & \\
Male & 70 & 59.8 & & $71-99$ \\
Age & & & $73.1(14.4)$ & 77 & \\
Discharged & 79 & 67.5 & & \\
Deceased & 38 & 32.4 & & \\
Length of hospital stay & & & $14.6(14.9)$ & 8 \\
Admitted to ICU & 17 & 14.5 & & \\
\hline
\end{tabular}

were considered for this analysis. The emergency percentage of discharges, deaths and ICU admissions was, respectively, $67.5 \%, 32.4 \%, 14.5 \%$, with an average length of stay of $14.6 \pm 14.9$ days.

The demographic, clinical and laboratory characteristics of datasets from the 117 consecutive patients admitted to the ER are reported in Table 2. All laboratory parameters considered in the present work were collected in the ED setting. Data entry was performed by a single attending physician blinded to the study procedures and protocol.

As described in the "Statistical analysis", variables identified were associated with at least one of the three outcomes originally considered (death, ICU, IV). Table 2 shows also the association of the best cutoff variables with the
Table 2 Demographic, clinical and laboratory variables associated with outcomes

\begin{tabular}{|c|c|c|c|c|c|}
\hline Variables & $N$ & Best cut-off & Sens (best cutoff) & Spec (best cutoff) & $p$ value \\
\hline Age & 117 & $>84$ & $38.0 \%$ & $80.3 \%$ & 0.220 \\
\hline Smoke (past) & 30 & & $22.2 \%$ & $47.6 \%$ & 0.106 \\
\hline Social distancing-R (above $1 \mathrm{~m}$ ) & 115 & & $33.3 \%$ & $77.6 \%$ & 0.192 \\
\hline CHD & 117 & & $8.0 \%$ & $79.1 \%$ & 0.056 \\
\hline Overweight & 82 & & $28.6 \%$ & $93.6 \%$ & 0.007 \\
\hline Dementia & 117 & & $30.0 \%$ & $79.1 \%$ & 0.259 \\
\hline Number of drugs & 115 & $\geq 6$ & $44.9 \%$ & $80.3 \%$ & 0.207 \\
\hline Not $100 \%$ self-sufficient & 115 & & $20.8 \%$ & $83.6 \%$ & 0.546 \\
\hline Confined in bed & 115 & & $22.9 \%$ & $91.0 \%$ & 0.038 \\
\hline Fever & 117 & & $78.0 \%$ & $26.9 \%$ & 0.547 \\
\hline Cough & 117 & & $34.0 \%$ & $59.7 \%$ & 0.487 \\
\hline Dyspnea & 117 & & $72.0 \%$ & $41.8 \%$ & 0.124 \\
\hline Tachypnoea & 107 & & $56.3 \%$ & $76.3 \%$ & 0.001 \\
\hline Cyanosis & 106 & & $21.3 \%$ & $96.6 \%$ & 0.004 \\
\hline Braden total & 101 & $<18$ & $84.8 \%$ & $63.6 \%$ & 0.000 \\
\hline $\mathrm{PO} 2$ & 54 & $<54.4$ & $70.0 \%$ & $88.2 \%$ & 0.000 \\
\hline $\mathrm{HCO} 3$ & 37 & $<23.0$ & $78.6 \%$ & $78.3 \%$ & 0.010 \\
\hline Lactate & 49 & $\geq 1.3$ & $70.0 \%$ & $72.4 \%$ & 0.003 \\
\hline $\mathrm{P} / \mathrm{F}$ & 76 & $<257$ & $74.3 \%$ & $70,7 \%$ & 0.000 \\
\hline $\mathrm{SpO} 2 \%$ & 102 & $<95$ & $85.7 \%$ & $51.7 \%$ & 0.010 \\
\hline PCR & 112 & $\geq 89.8$ & $68.8 \%$ & $70.3 \%$ & 0.000 \\
\hline PCT & 90 & $\geq 0.18$ & $66.7 \%$ & $76.5 \%$ & 0.000 \\
\hline Fibrinogen & 103 & $<511$ & $51.2 \%$ & $68.9 \%$ & 0.182 \\
\hline LDH & 97 & $\geq 439$ & $61.0 \%$ & $87.5 \%$ & 0.000 \\
\hline AST & 95 & $\geq 44$ & $61.0 \%$ & $74.1 \%$ & 0.004 \\
\hline Creat & 111 & $\geq 1.57$ & $33.3 \%$ & $84.1 \%$ & 0.031 \\
\hline CPK & 105 & $\geq 84$ & $83.3 \%$ & $43.9 \%$ & 0.003 \\
\hline CPAP & 117 & & $58.0 \%$ & $79.1 \%$ & 0.000 \\
\hline
\end{tabular}

CHD, coronary heart disease; PCR, polymerase chain reaction; PCT, procalcitonin; LDH, lactate dehydrogenase; AST, aspartate aminotransferase; CPK, creatine phosphokinase; CPAP, continuous positive airway pressure 
composite in-hospital death OR ICU admission outcome (in brackets the best cutoffs identified for numerical variables, with ROC analysis).

As displayed in Table 3, three variables (Braden score $<18, \mathrm{LDH} \geq 439 \mathrm{U} / \mathrm{L}$ and $\mathrm{P} / \mathrm{F}<257$ ) were identified in the multiple logistic regression analysis as significantly associated with the composite outcome (Table 3). The threeitem indicator was named Braden-LDH-HorowITZ (BLITZ) assessment.

The mathematical algorithm used to calculate BLITZ is as follows:

Prob $($ ICU OR Death $)=1 /(1+\exp (-\mathrm{k}))$

$\mathrm{k}=-2.192+1.358 *(1$ if $\mathrm{BRADEN} \geq 18 ; 0$ if BRADEN $<18)+2.261 *(1$ if $\mathrm{LDH} \geq 439 ; 0$ if $\mathrm{LDH}<439)+1.258 *(1$ if $\mathrm{PaO} 2 / \mathrm{FiO} 2<257 ; 0$ if $\mathrm{PaO} 2 /$ $\mathrm{FiO} 2 \geq 257$ ).

with -2.192 being the constant; $1.358 ; 2.261 ; 1.258$ being the $\beta$-coefficients from logistic regression for the respective investigated factors. Starting from the logistics equation, we proceeded to calculate the risks (probability) of adverse outcome (ICU or death). The risk for adverse outcomes (ICU OR death) with a scoring from one to three, with relevant differences among relative weight of each item, is presented in Table 4.

\section{Discussion}

In this retrospective analysis of a real-world sample of COVID-19 patients admitted to the ED, we disclosed a significant association between a feasible, rapid BLITZ assessment including Braden value below 18, blood LDH concentration above $439 \mathrm{U} / \mathrm{L}$ as well as a P/F below 257 and adverse outcomes including death and ICU admission. The BLITZ-parameters showed good discriminatory power and accuracy in predicting the relevant endpoints chosen irrespective of chronological age. To date, there is no single prognostic or therapeutic algorithm able to univocally guide clinical decisions during the pandemic phases preceding and accompanying the vaccination [10]. This lack, mainly driven by the focus of existing stratification tools exclusively on chronological age, organ function and morbidity, hinders the effective triaging with advancing age. Indeed, there is mounting evidence that multidimensional frailty beyond chronological age and organ specific function is a major driver of outcomes and life trajectories after SARS-CoV-2 infection [2, 11]. Accordingly, a number of scores and early warning prognostic tools [12-16] have been recently developed to determine the risk of death in the ED setting. However, within this frame, BLITZ profiles itself through its highest clinimetric properties and inclusion of social and functional aspects addressed by the Braden scale. This underlines the need of a paradigm shift towards the attention for person-centered factors beyond-organ medicine also in urgent settings [10,17], where the feasibility of multidimensional prognostic tools based on more comprehensive assessments has been demonstrated [17].

The paradigm shift suggested here is that BLITZ offers a feasible, multidimensional way-even though far from a comprehensive assessment-based prognostic tool-to capture person-centered risk of COVID-19-related poor outcomes beyond infection parameters. This is highly relevant for clinical practice as, due to the accelerating expansion in number of old and very old persons, a progressively larger percentage of the hospitalized patients are older and multimorbid; reliable, feasible risk indicators taking into account the overall functions of the person beyond illnesses and age, applicable across a wide range of healthcare settings and not requiring high-performance medicine, are urgently needed. On the contrary, advanced chronological age and
Table 3 Composite outcomeidentified variables of the BLITZ estimated by multiple logistic regression

\begin{tabular}{lllllll}
\hline Source & Beta coeff & Standard error & $P$ value & Odds ratio & OR 95\% CI lower & OR 95\% CI upper \\
\hline Intercept & -2192 & 0.423 & 0.316 & & & \\
BRADEN $<18$ & 1358 & 0.502 & 0.007 & 3.888 & 1.453 & 10.405 \\
LDH $\geq 439$ & 2261 & 0.548 & $<0.00019 .593$ & 3.278 & 28.070 \\
P/F $<257$ & 1258 & 0.480 & 0.008 & 3.517 & 1.373 & 9.010 \\
\hline
\end{tabular}

Table 4 Estimate of the risk of adverse outcome for the different combinations of the 3 risk factors included in BLITZ $(1=$ yes, $0=$ no $)$

\begin{tabular}{|l|l|l|l|l|l|l|l|l|}
\hline BRADEN $<18$ & 0 & 0 & 1 & 0 & 1 & 0 & 1 & 1 \\
\hline LDH $\geq 439$ & 0 & 0 & 0 & 1 & 0 & 1 & 1 & 1 \\
\hline PaO2/FiO2 <257 & 0 & 1 & 0 & 0 & 1 & 1 & 0 & 1 \\
\hline & & & & & & & & \\
\hline Risk of death OR ICU & $10.1 \%$ & $28.2 \%$ & $30.3 \%$ & $51.7 \%$ & $60.4 \%$ & $79.0 \%$ & $80.6 \%$ & $93.6 \%$ \\
\hline
\end{tabular}


multimorbidity are currently given highest priority for triaging during the present pandemic [8]. However, in our analysis, chronological age does not appear to play a major role for poor outcome prediction. Interestingly, among the several variables included in the analysis, those captured by the Braden Scale have reached highest clinimetric threshold (Table 3). The scale is feasible, being performed in few minutes by ED nurses. The Braden scale addresses domains beyond-organ function and includes functional, cognitive and nutritional aspects that are typically known to influence patients' trajectories during and after hospitalization [18, 19]. Our observation is in line with recent studies showing a critical role of frailty and functional status in determining COVID-19-related trajectories [2, 11, 12, 20-25].

However, neither is frailty consistently included among potential stratification strategies, nor it is systematically assessed in clinical routine, especially in emergency settings. A decisive instrument to disentangle complexity of clinical pictures in advanced age is the comprehensive geriatric assessment (CGA) [26, 27]. A metanalysis of 29 randomized controlled trials conducted in over 14,000 older patients have shown that the CGA is highly effective in improving diagnosis and management [28]. Recent studies employing CGA-based innovative, feasible tools for the assessment of multidimensional, individualized prognosis clearly showed that the latter disclose critical factors for trajectories which go beyond organ-center medicine and chronological age [29-31] and apply also during the ongoing pandemic $[11,23]$. Of note, in our analysis the Braden scale but not the Conley scale-which addresses physical factors only-reached and surpassed the predictive power of the $\mathrm{P} / \mathrm{F}$ or of the LDH levels, supporting the knowledge that functions escaping diagnostics in usual care influence the ability to thrive in advanced age. For instance, nutrition is known as an essential actor of patient's recovery and resistance against bacterial and viral infections [32]. As other factors other than an approximative estimation of frailty might substantially influence disease course, like resiliency, nutritional status, polypharmacy and social condition, the use of structured prognostic instruments appears to be highly recommended to avoid vague "clinical reasonableness", ageism and inadequate management. Simple and reliable tools for the estimation of the prognosis of the older patients are needed to tailor clinical management of older patients.

Although the analysis conveys the strengths of high accuracy of the assessment as well as clear feasibility and reallife application in a catchment area of over 42,000 patients per year during the pandemic, some limitations must be acknowledged, first, the retrospective nature of the analysis of a relatively sample of data. However, the stratification advantage of a multidimensional approach was given highest priority at this stage to provide emergency physicians with a rapid tool for enabling beyond-organ urgent clinical decisions and tailored interventions. A further limitation of the present analysis is that it has been performed after ventilation allocation. However, the strong power of BLITZ, showing that a pathologic Braden score almost doubles the risk of poor outcomes compared to the largely accepted parameters LDH and Horowitz index, suggests the likelihood for generalizability to different allocation phases as well as to larger populations and more settings.

\section{Conclusion}

In conclusion, our analysis shows that the BLITZ model, which includes P/F, LDH and the Braden scale as a measure of overall status of the person, but not chronological age, is able to predict with highest accuracy the probability of patients to suffer from the main COVID-19-related adverse outcomes such as admission to ICU and death.

Further prospective investigations are needed to assess whether this prediction model can be validated in larger cohorts in emergency as well as in other settings.

Acknowledgements The authors wish to thank ASIAOnlus for their contribution in the development of the BLITZ assessment

\section{Declarations}

Conflict of interest The authors declare that they have no conflict of interest.

Ethical approval For this study, at the beginning of the COVID pandemic, formal written consent was not asked.

Animal and human rights This study is based on data retrieved at patients' admission at the beginning of the pandemic. The analyses were performed retrospectively.

Informed consent For this type of study, formal consent is not required.

\section{References}

1. Zhou F, Yu T, Du R, Fan G, Liu Y, Liu Z et al (2020) Clinical course and risk factors for mortality of adult inpatients with COVID-19 in Wuhan, China: a retrospective cohort study. Lancet 395:1054

2. Hewitt J, Carter B, Vilches-Moraga A, Quinn TJ, Braude P, Verduri A et al (2020) The effect of frailty on survival in patients with COVID-19 (COPE): a multicentre, European, observational cohort study. Lancet Public Health 5(8):e444-e451

3. Weiss P, Murdoch DR (2020) Clinical course and mortality risk of severe COVID-19. Lancet 395(10229):1014-1015

4. Bartoli A, Gabrielli F, Alicandro T, Nascimbeni F, Andreone P (2021) COVID-19 treatment options: a difficult journey between failed attempts and experimental drugs. Intern Emerg Med 16:281

5. Lopez-Bueno R, Calatayud J, Ezzatvar Y, Casajus JA, Smith L, Andersen LL et al (2020) Association between current physical 
activity and current perceived anxiety and mood in the initial phase of COVID-19 confinement. Front Psychiatry 11:729

6. Chen T, Wu D, Chen H, Yan W, Yang D, Chen G. Clinical characteristics of 113 deceased patients with coronavirus disease 2019: retrospective study [published online March 26, 2020]. BMJ. 2020.

7. Sattar N, McInnes IB, McMurray JJ (2020) Obesity a risk factor for severe COVID-19 infection: multiple potential mechanisms. Circulation 1:4

8. Izcovich A, Ragusa MA, Tortosa F, Lavena Marzio MA, Agnoletti C, Bengolea A et al (2020) Prognostic factors for severity and mortality in patients infected with COVID-19: a systematic review. PLoS ONE 15(11):e0241955

9. Kang H (2013) The prevention and handling of the missing data. Korean J Anesthesiol 64(5):402-406

10. Polidori MC, Sies H, Ferrucci L, Benzing T (2021) COVID19 mortality as a fingerprint of biological age. Ageing Res Rev 67:101308

11. Pilotto A, Azzini M, Cella A, Cenderello G, Castagna A, Pilotto A et al (2021) The multidimensional prognostic index (MPI) for the prognostic stratification of older inpatients with COVID-19: a multicenter prospective observational cohort study. Arch Gerontol Geriatrics. 95:104415

12. Covino M, Sandroni C, Santoro M, Sabia L, Simeoni B, Bocci MG et al (2020) Predicting intensive care unit admission and death for COVID-19 patients in the emergency department using early warning scores. Resuscitation 156:84-91

13. Haimovich AD, Ravindra NG, Stoytchev S, Young HP, Wilson FP, van Dijk D et al (2020) Development and validation of the quick COVID-19 severity index: a prognostic tool for early clinical decompensation. Ann Emerg Med 76(4):442-453

14. Knight SR, Ho A, Pius R, Buchan I, Carson G, Drake TM, et al. Risk stratification of patients admitted to hospital with covid-19 using the ISARIC WHO Clinical Characterisation Protocol: development and validation of the 4C Mortality Score. Bmj. 2020;370.

15. Liang W, Liang H, Ou L, Chen B, Chen A, Li C et al (2020) Development and validation of a clinical risk score to predict the occurrence of critical illness in hospitalized patients with COVID19. JAMA Intern Med 180(8):1081-1089

16. Smith GB, Prytherch DR, Meredith P, Schmidt PE, Featherstone PI (2013) The ability of the National Early Warning Score (NEWS) to discriminate patients at risk of early cardiac arrest, unanticipated intensive care unit admission, and death. Resuscitation 84(4):465-470

17. Rarek MP, Meyer AM, Pickert L, Pilotto A, Benzing T, Burst V et al (2020) The prognostic signature of health-related quality of life in older patients admitted to the emergency department: a 6-month follow-up study. Aging Clin Exp Res. https://doi.org/10. 1007/s40520-020-01732-8

18. Marengoni A, Zucchelli A, Vetrano DL, Armellini A, Botteri E, Nicosia F et al (2020) Beyond chronological age: frailty and multimorbidity predict in-hospital mortality in patients with coronavirus disease 2019. J Gerontol A Biol Sci Med Sci 76:e38

19. Polidori MC. Geriatrics' turning point. Springer; 2019.
20. Laosa O, Pedraza L, Álvarez-Bustos A, Carnicero JA, RodriguezArtalejo F, Rodriguez-Mañas L (2020) Rapid assessment at hospital admission of mortality risk from COVID-19: the role of functional status. J Am Med Directors Association 21(12):1798-802. e2

21. Hwang J, Ryu H-S, Kim HA, Hyun M, Lee JY, Yi H-A (2020) Prognostic factors of COVID-19 infection in elderly patients: a multicenter study. J Clin Med 9(12):3932

22. Heras E, Garibaldi P, Boix M, Valero O, Castillo J, Curbelo Y, et al. COVID-19 mortality risk factors in older people in a longterm care center. Eur Geriatric Med. 2020; 1-7.

23. Becerra-Muñoz VM, Núñez-Gil IJ, Eid CM, García Aguado M, Romero R, Huang J et al (2021) Clinical profile and predictors of in-hospital mortality among older patients hospitalised for COVID-19. Age Ageing 50(2):326-334

24. Covino M, De Matteis G, Polla DDA, Santoro M, Burzo ML, Torelli E et al (2021) Predictors of in-hospital mortality AND death RISK STRATIFICATION among COVID-19 PATIENTS aged $\geq 80$ YEARs OLD. Arch Gerontol Geriatrics. 95:104383

25. Aliberti MJR, Covinsky KE, Garcez FB, Smith AK, Curiati PK, Lee SJ et al (2021) A fuller picture of COVID-19 prognosis: the added value of vulnerability measures to predict mortality in hospitalised older adults. Age Ageing 50(1):32-39

26. Rubenstein L, Siu A, Wieland D (1989) Comprehensive geriatric assessment: toward understanding its efficacy. Aging Clin Exp Res $1(2): 87-98$

27. Pilotto A, Cella A, Pilotto A, Daragjati J, Veronese N, Musacchio C et al (2017) Three decades of comprehensive geriatric assessment: evidence coming from different healthcare settings and specific clinical conditions. J Am Med Dir Assoc 18(2):192 e1-e11

28. Ellis G, Gardner M, Tsiachristas A, Langhorne P, Burke O, Harwood RH et al (2017) Comprehensive geriatric assessment for older adults admitted to hospital. Cochrane Database Syst Rev 9:CD006211

29. Meyer AM, Becker I, Siri G, Brinkkotter PT, Benzing T, Pilotto A et al (2019) New associations of the Multidimensional Prognostic Index. Z Gerontol Geriatr 52(5):460-467

30. Meyer AM, Becker I, Siri G, Brinkkotter PT, Benzing T, Pilotto A et al (2020) The prognostic significance of geriatric syndromes and resources. Aging Clin Exp Res 32(1):115-124

31. Meyer AM, Siri G, Becker I, Betz T, Bodecker AW, Robertz JW et al (2019) The multidimensional prognostic index in general practice: one-year follow-up study. Int J Clin Pract 73:e13403

32. Schuetz P, Fehr R, Baechli V, Geiser M, Deiss M, Gomes F et al (2019) Individualised nutritional support in medical inpatients at nutritional risk: a randomised clinical trial. Lancet 393(10188):2312-2321

Publisher's Note Springer Nature remains neutral with regard to jurisdictional claims in published maps and institutional affiliations. 